\title{
Proposta de modelo mediacional entre suporte organizacional, capital psicológico e saúde geral em enfermeiros de um hospital público
}

\author{
The mediation model between organizational support, \\ psychological capital and general health in nurses \\ from a public hospital
}

\section{Propuesta de un modelo mediacional entre soporte organizacional, capital psicológico y salud general en enfermeros de un hospital público}

\author{
Nilton Soares Formiga* \\ Gabriela Aguiar Pereira ${ }^{* *}$ \\ Ionara Dantas Estevam***
}

\section{Resumo}

Os constructos do Capital Psicológico e Suporte Organizacional são áreas do conhecimento que propõem o resgate do valor das pessoas e suas implicações no ambiente das empresas. O presente estudo tem como objetivo analisar a relação entre suporte organizacional, capital psicológico positivo no trabalho e saúde geral em enfermeiros de um hospital público na cidade de Natal-RN. Tratou-se de uma pesquisa quantitativa, e, para coleta de dados, foram utilizados 04 instrumentos: Questionário de caracterização sociodemográfico e laboral; Escala de Percepção de Suporte Organizacional, Escala de Capital Psicológico Positivo no Trabalho e o Questionário de Saúde Geral, aplicados a 101 enfermeiros. Por meio do pacote estatístico SPSSWIN 24.o e AMOS 22, realizaram-se as análises estatísticas descritivas, correlação de Pearson,

\footnotetext{
* Universidade Potiguar, RN, Brasil. E-mail: nsformiga@yahoo.com

** Universidade Potiguar, RN, Brasil. E-mail: gabyyaguiar@hotmail.com

*** Universidade Potiguar, RN, Brasil. E-mail: ionara.dantas@unp.br
} 
teste $t$ de Student, alfas e verificação do modelo teórico. Observou-se que a associação entre o suporte organizacional e questionário de saúde geral não foi significativa, refutando a hipótese inicial. Gerou-se um modelo alternativo, hipotetizando uma perspectiva mediacional. Nesta, a variável suporte organizacional associou-se positivamente ao capital psicológico, e este ao QSG. Conclui-se que o modelo alternativo apresentou melhores resultados, demonstrando que, quando o capital psicológico atua como mediador entre o suporte organizacional e a saúde geral dos enfermeiros, é possível obter melhores níveis de saúde.

Palavras-chave: Capital Psicológico; Suporte Organizacional; Saúde Geral; Enfermeiros.

\section{Abstract}

The Psychological Capital and Organizational Support constructs are areas of knowledge which suggest the redemption of people's value and their implications over the companies' environment. The present study aims to analyze the relationship between organizational support, positive psychological capital and general health in nurses from a public hospital in the city of Natal, state of Rio Grande do Norte, Brazil. The study quantitative field research, data collection used o4 instruments: a social-demographic and labor characterization questionnaire; the Perceived Organizational Support Scale; the Positive Psychological Capital Scale at Work, and the General Health Questionnaire, applied to 101 nurses. Through the SPSSWIN 24.O and AMOS 22 statistical package, descriptive statistical analysis was carried out, the Pearson correlation, the Student's t-test, alphas and the theoretical model validation. It was observed that the association between organizational support and QSG was not significant, refuting the initial hypothesis. An alternative model was generated, hypothesizing a mediation perspective, in this, the organizational support variable was positively associated with psychological capital, and this with QSG. The research concludes that the the alternative model presented better results, shows that when the psychological capital acts as a mediator between the organizational support and the nurses'general health, it is possible to reach better health levels.

Keyword: Psychological Capital; Organizational Support, General Health; Nurses.

\section{Resumen}

Los constructos del Capital Psicológico y Soporte Organizacional son áreas del conocimiento que proponen el rescate del valor de las personas y sus implicaciones en el ambiente de las empresas. El presente estudio tiene como objetivo analizar la relación entre soporte organizacional, capital psicológico positivo en el trabajo y salud general en los enfermeros de un hospital público 
en la ciudad de Natal-RN. Se trata de una investigación cuantitativa, para la recolección de datos se utilizaron 04 instrumentos: Cuestionario de caracterización sociodemográfica y laboral; Escala de percepción de soporte organizacional; Escala de capital psicológico positivo en el trabajo y Cuestionario de salud general, aplicados a 101 enfermeros. A través del paquete estadístico SPSSWIN 24.O y AMOS 22, se llevaron a cabo los análisis estadísticos descriptivos, correlación de Pearson, prueba t de Student, alphas y verificación del modelo teórico. Se observó que la asociación entre apoyo organizacional y CSG no fue significativa, refutando la hipótesis inicial. Se generó un modelo alternativo, hipotetizando una perspectiva mediacional, en este, la variable de apoyo organizacional se asoció positivamente con el capital psicológico, y este con CSG. Se concluye que el modelo alternativo mostró mejores resultados, demostrando que cuando el capital psicológico actúa como mediador entre el apoyo organizacional y la salud general de las enfermeras, es posible obtener mejores niveles de salud.

Palabras clave: Capital Psicológico; Soporte Organizacional; Salud General; Enfermeros.

\section{INTRODUÇÃO}

As transformações ocorridas no mundo do trabalho e da empregabilidade nos últimos vinte anos têm sido um fenômeno que repercute nos aspectos econômicos, políticos e comportamentais. Tais eventos centram-se nos atores do trabalho (isto é, os próprios trabalhadores), parte prioritária no processo da manutenção da subjetividade individual e coletiva referente ao sentido e significado do trabalho na sociedade (Antunes, 2009; Csikszentmihalyi, 2004; Barbosa, 2014; Scorsolini-Comin \& Santos, 2010). É possível que tais subjetividades impactem na economia, política, cultura e na relação sociedade-organização-trabalho, tornado precário e frágil o binômio saúde-trabalho, infuenciando no bem-estar físico, psicológico e social do trabalhador. Em decorrência deste cenário, os profissionais na área da saúde estão entre as categorias laborais mais adoecidas, identificando problemas de transtorno mental, físico e, consecutivametne, de afastamento do trabalho por tempo limitado (Zanelli, Borges-Andrade \& Bastos, 2004; Leite \& Nogueira, 2017).

A fim de compreender os processos do trabalho, sua relação e seus efeitos nas pessoas, pesquisadores na área das ciências humanas e sociais 
aplicadas a organização e ao trabalho sugerem que, ao identificar e avaliar essas dimensões, é possível verificar a influência direta ou indireta, na saúde do trabalhador (Antunes, 2009; Avey, Luthans, \& Jensen, 2009; Bendassoli, 2011). Neste contexto, o trabalhador é capaz de desenvolver meios de adaptação aos processos mais dinâmicos de produção, bem como recursos psicológicos destinados às tomadas de decisão acerca das dificuldades no trabalho, sobretudo, aqueles que trabalham na área da saúde (Torres, Chagas, Moreira, Barreto, \& Rodrigues, 2013). No estudo de Formiga e Guimarães (2019), esta afirmação foi corroborada; estes autores avaliaram o transtorno emocional em médicos no Rio Grande do Norte e observaram que os profissionais que desenvolveram um capital psicológico positivo apresentaram escores mais baixo na ansiedade, depressão e estresse. Da mesma forma, aquele profissional que investiu no cuidado com sua saúde mental (isto é, faz terapia e/ou tratamento psiquiátrico) não apenas apresentou um maior escore no capital psicológico, como também, os escores na avaliação do transtorno emocional foram muito menores.

Desta forma, vem se observando um número crescente de estudos destinados à avaliação dos impactos e os custos que o adoecimento tem produzido no trabalho e como este é capaz de influenciar a saúde de profissionais enfermeiros (Murofuse \& Marziale, 2005; Chaves, Ramos, \& Figueiredo, 2011; Lino, Nora, Lino, \& Furtado, 2012; Fabri et al., 2018). Estes, entre outros estudos, revelam que os enfermeiros estão expostos a riscos ocupacionais físicos, químicos e biológicos e, também, ao sofrimento psíquico, pois lidam constantemente com a fragilidade na gravidade da saúde dos outros e com a morte (Castro \& Farias, 2008; Brito, Pena, Gomes, Souto, \& Pires, 2012; Gomes, 2017; Barros \& Honório, 2015). Com isso, o adoecimento destes profissionais poderá se associar a situações muito mais complexas, como, por exemplo, a criação de vínculos afetivos entre profissional e paciente, a escassez de funcionários, o acúmulo de tarefas e sobrecarga de trabalho, turno noturno de trabalho, relacionamento com usuários e familiares, conflito e ambiguidade de papéis, baixa participação nas decisões, injustiças nas relações laborais e conflitos entre os colegas de trabalho, interferindo no bem-estar deles, especialmente, devido a falta de estratégias de enfrentamento que pudessem intervir nos desgastes da 
saúde laboral (Galindo, Feliciano, Lima, \& Souza, 2012; Lorenz, Benatti, \& Sabino, 2010; Fabri et al., 2018). Todavia, o fenômeno do adoecimento não é algo que ocorre no vazio, podendo se associar à própria condição da organização relativa ao apoio e suporte oferecido, bem como, à capacidade de o profissional desenvolver e manter o equilíbrio emocional. Desta maneira, alguns construtos da psicologia positiva e do comportamento organizacional positivo podem ser úteis para compreender este fenômeno no ambiente de trabalho. No presente estudo, serão contempladas as variáveis do suporte organizacional e do capital psicológico positivo e o quanto elas influenciam a saúde geral do enfermeiro.

Os construtos supracitados propõem resgatar a subjetividade e as implicações no ambiente das empresas, trabalhando a ação motivada e as forças psíquicas dos trabalhadores, os quais podem guiar os seus comportamentos para um melhor desempenho e produtividade na organização (Siqueira, 1995; Caesens, Stinglhamber, \& Ohana, 2016), para o florescimento e o funcionamento saudável do indivíduo, propiciando os aspectos psicológicos, biológicos e sociais positivos em experiências individuais, grupais e culturais (Siqueira \& Padovan, 2008; Siqueira, 2014). Desta forma, o Capital Psicológico Positivo (PsyCap) é concebido como um estado de desenvolvimento psicológico positivo, relacionado a resultados e desempenhos no trabalho, a saber: comprometimento, satisfação, perseverança e comportamento organizacional positivo (Luthans, Luthans, \& Luthans, 2004).

Indivíduos com capital psicológico elevado possuem características do tipo: ter confiança para assumir e colocar o esforço necessário para ter sucesso em tarefas desafiadoras (autoeficácia), fazer uma atribuição positiva sobre ter sucesso agora e no futuro (otimismo), ser perseverante perante as metas e, quando necessário, redirecionar os caminhos para alcançar o sucesso (esperança) e, quando abalado por problemas e adversidades, sustentar e recuperar, ir mais longe e mais além para alcançar o sucesso (resiliência) (Luthans, Youssef \& Avolio, 2007). Por esse motivo, Avey, Reichard, Luthans e Mhatre (2011) explicam que atitudes e comportamentos positivos dos colaboradores no ambiente organizacional são influenciados pelo capital psicológico, associando positivamente a performance, a 
satisfação, o comprometimento organizacional, a inovação, a felicidade etc. Por outro lado, indivíduos com um baixo capital psicológico desenvolvem atitudes indesejáveis no trabalho como cinismo, estresse, turnover e ansiedade. Apesar de tal valorização do capital psicológico positivo, este não ocorreria de forma isolada na dinâmica interna da organização, podendo se associar à percepção do suporte organizacional do próprio trabalhador no seu ambiente laboral.

Segundo Siqueira (2014), o suporte organizacional é a capacidade de oferecer sustentação, apoio e proteção ao trabalhador, destinada à inibição e controle d adoecimento físico e emocional deles na organização. Foram Eisenberger, Huntington, Hutchison e Sowa (1986) que desenvolveram a noção e o conceito do suporte organizacional, compreendido como as crenças globais desenvolvidas pelo empregado sobre a extensão em que a organização valoriza as suas contribuições e cuida do seu bem-estar e, sob tal conceito, organizaram uma medida psicológica para trabalhadores. Sendo assim, o suporte organizacional tem sua base de função, estrutura e organização nas expectativas de troca e benefício mútuo do trabalhador com sua organização (Oliveira-Castro, Pilati, \& Borges-Andrade, 1999; Chong, White, \& Prybutok, 2001; Paschoal, 2008; Neves \& Eisenberger, 2014). Associado à concepção de bem-estar, tal construto está diretamente ligado à ideia de prazer e felicidade, com foco na experiência de expressão pessoal e de autorrealização, na satisfação no ambiente organizacional, nas condições de trabalho que favoreçam o benefício ao trabalhador e seu ambiente, contribuindo com a qualidade de vida, o baixo estresse e a boa saúde mental (Tamayo \& Tróccoli, 2002; Paschoal, Torres, \& Porto, 2010; Formiga, Freire, \& Fernandes, 2019). Considerando a relevância destes pressupostos teóricos, o presente estudo pretende compreender as vivências de enfermeiros no exercício de sua atividade, verificando a relação interdependente entre suporte organizacional, capital psicológico positivo no trabalho e saúde geral em enfermeiros de um hospital público na cidade de Natal-RN. 


\section{MÉTODO}

Trata-se de um estudo descritivo, exploratório e correlacional, de abordagem quantitativa, envolvendo profissionais na área da saúde de um hospital público na cidade de Natal-RN. No enfoque quantitativo é permitida a coleta sistemática de informações numéricas, mediante condições de controle analisadas por meio de estatística (Polit \& Beck, 2011).

\section{Participantes}

Para o cálculo da amostra utilizou-se o pacote estatístico GPower 3.1, o qual tem como objetivo verificar a significância do 'n' amostral para a presente estudo; este programa é software destinado a calcular o poder estatístico (isto é, o teste de hipótese), tendo como base não apenas o 'n' necessário para a pesquisa, mas, também, o tipo de cálculo a ser realizado (Faul, Erdfelder, Lang \& Buchner, 2007). Considerando uma probabilidade de 95\% ( $\mathrm{p}<0,05)$, a magnitude do efeito amostral ( $r \geq 0,50)$ e um padrão de poder hipotético $(\pi \geq 0,80)$, observou-se, a partir desses critérios, que uma amostra mínima de 80 participantes seria suficiente (tendo como indicadores estatísticos: $t \geq 1,98 ; \pi=0,91 ; \mathrm{p}<0,05)$. Apesar o ' $\mathrm{n}$ ' indicado, considerando o método de coleta bola de neve, com amostra intencional, obteve-se, na coleta final, uma amostra composta por 101 enfermeiros de um hospital público na cidade de Natal-RN / Brasil.

Quanto aos critérios de inclusão, considerou-se o sujeito que esteja empregado por mais de um ano, ativo no seu setor de trabalho, no Regime Jurídico Único - RJU ou CLT, ser da área da saúde no hospital onde será feita a coleta de dados e ter jornada de trabalho entre 20 e 40 horas semanais. O estudo foi desenvolvido na Maternidade Escola Januário Cicco (MEJC), pertencente ao Complexo de Saúde da Universidade Federal do Rio Grande do Norte - UFRN, localizado na região metropolitana do município de Natal/RN, administrada pela Empresa Brasileira de Serviços Hospitalares (EBSERH).

Nesta pesquisa observaram-se as seguintes características sociodemográficas: 101 enfermeiros, todos funcionários do hospital público 
na cidade de Natal-RN, a maioria (64\%) integrante a EBSERH (Empresa Brasileira de Serviços Hospitalares). A amostra foi composta por 95\% de mulheres, com uma média etária de 40,16 anos; 63\% eram casados, 55\% apresentaram uma renda entre 6 e 7 salários-mínimos. Em relação à informação sobre o profissionalismo dos respondentes: 74\% tinha especialização na área, 66\% atua por um a cinco anos, com 19\% destes trabalhando no setor da UTI neonatal; 67\% possuem carga horária semanal de 36 horas e 80\% dos respondentes não exerce função de gestão. Referente à saúde laboral, 61\% afirma não ter estado de licença por motivo de saúde e 95\% deles relata não ter sofrido nenhum acidente no local de trabalho.

\section{Instrumentos para coleta de dados}

Foram utilizados os seguintes instrumentos:

\section{Escala de Percepção de Suporte Organizacional (EPSO)}

- Trata-se de uma escala composta por 9 itens $(\mathrm{SO} 1=$ Esta empresa ignoraria qualquer reclamação de minha parte; SO2 = Esta empresa não considera meus interesses quando toma decisões que me afetam; $\mathrm{SO}_{3}=$ É possível obter ajuda desta empresa quando tenho um problema; $\mathrm{SO}_{4}$ = Esta empresa realmente preocupa-se com meu bem-estar; $\mathrm{SO}_{5}=$ Esta empresa estaria disposta a ampliar suas instalações para me ajudar a utilizar minhas melhores habilidades no desempenho do meu trabalho; SO6 = Esta empresa está pronta a ajudar-me quando eu precisar de um favor especial; $\mathrm{SO}_{7}$ = Esta empresa preocupa-se com minha satisfação com o trabalho; SO8 = Esta empresa preocupa-se mais com seus lucros do que comigo e $\mathrm{SO} 9$ = Esta empresa tenta fazer com que meu trabalho seja o mais interessante possível), desenvolvida por Eisenberger et al. (1986) e adaptada e validade para o contexto brasileiro por Siqueira (1995). Esta medida tem por objetivo avaliar o quanto as pessoas (que trabalham em organizações públicas e/ou privadas) percebem que a empresa tem uma preocupação com o bem-estar do empregado. Para mensurar o construto, o respondente deveria indicar a sua resposta, marcando com um $\mathrm{X}$ ou um círculo em uma escala de sete pontos que variava de 1 (discordo totalmente) a 7 (concordo totalmente). Em relação à confiabilidade da escala, no pioneiro estudo de Siqueira (1995) 
foi observado que essa medida apresentou um alfa de 0,86 , revelando uma consistência interna na mensuração do construto. Adicionalmente, em uma amostra com trabalhadores brasileiros, Formiga, Fleury e Souza (2014) observaram a consistência da estrutura fatorial da escala, com indicadores psicométricos que garantiram a organização fatorial proposta por Siqueira (1995) $\left(X^{2} / \mathrm{gl}=1,42, \mathrm{RMR}=0,02, \mathrm{GFI}=0,99\right.$, AGFI $=0,97, \mathrm{CFI}=0,99$, $\mathrm{TLI}=0,99$ e RMSEA $=0,03$ ).

\section{Escala de Capital Psicológico Positivo no Trabalho (ECPP) -}

Trata-se de um instrumento elaborado por Luthans et al. (2007). Neste estudo utilizou-se uma forma reduzida, com 12 itens, desenvolvida por Viseu et al. (2012) para o contexto Português, em Portugal. Ela é composta por itens que descrevem para os respondentes um estado psicológico positivo, com o objetivo de enfrentar e empregar o esforço necessário para alcançar sucesso em tarefas desafiadoras. De acordo com os autores, a escala é formada por quatro fatores, sendo três itens relacionados à autoeficácia (AE 1 = Sinto confiança em representar minha área de trabalho em reuniões com a gestão; AE 2 = Sinto confiança em contribuir para discussões sobre a estratégia da minha empresa/instituição e AE 3 = Sinto confiança em apresentar informações a um grupo de colegas); quatro à esperança (ESPER 1 = Se sentir que estou bloqueado no trabalho, penso em muitas formas de solucionar; ESPER 2 = Neste momento, penso que tenho bastante sucesso no trabalho; ESPER 3 = Consigo pensar em muitas formas de alcançar os meus objetivos de trabalho atuais e ESPER 4 = Nesta altura, estou a atingir os objetivos de trabalho que defini para mim próprio); três à resiliência (RESILI 1 = Posso contar só comigo próprio no trabalho se tiver de ser; RESILI 2 = Habitualmente lido com as questões estressantes com facilidade e RESILI 3 = Consigo lidar com alturas difíceis no trabalho porque já passei por dificuldades antes); e dois ao otimismo (OTIMI $1=$ Vejo sempre o lado bom das coisas sobre o meu trabalho e OTIMI $2=$ Sou otimista sobre o que me vai acontecer no futuro sobre o trabalho). O sujeito deveria responder numa escala tipo Likert, de seis pontos, o grau de concordância com cada itens, variando de 1 (discordo fortemente) a 6 (concordo fortemente). No estudo desenvolvido por Formiga, Viseu e Jesus (2014) para o contexto brasileiro, a fim de validar a medida para o referido contexto 
com amostras de trabalhadores de organizações públicas e privadas, os autores observaram, através da análise fatorial confirmatória, indicadores psicométricos confiáveis $\left(X^{2} / \mathrm{gl}=1,32, \mathrm{RMR}=0,05, \mathrm{GFI}=0,98, \mathrm{AGFI}=\right.$ 0,95, CFI = 0,99, TLI $=0,99$ e RMSEA $=0,03)$, confirmando a estrutura tetrafatorial da ECPP em Portugal (Luthans et al, 2007; Viseu et al., 2012).

Questionário de Saúde Geral (QSG-12) - Este instrumento é uma versão reduzida do QSG-60, de Goldberg e Williams, e adaptado ao Brasil por Pasquali, Gouveia, Andriola, Miranda e Ramos (1994; Gouveia et al., 2003). É composto por 12 itens (QSG 1 = Você tem conseguido se concentrar bem naquilo que faz; QSG 2 = Você tem sentido que está desempenhando um papel útil na vida; QSG 3 = Você tem se sentido capaz de tomar decisões; QSG 4 = Você tem realizado com satisfação suas atividades normais do dia-a-dia; QSG 5 = Você se sente razoavelmente feliz, considerando todas as circunstancias; QSG 6 = Você tem sido capaz de enfrentar seus problemas adequadamente; QSG 7 = Você tem se sentido constantemente esgotado e sob pressão; QSG 8 = Você tem tido a sensação de que não pode superar as dificuldades; QSG 9 = Você tem se sentido infeliz ou deprimido? QSG 10 = Você tem perdido a confiança em si mesmo; QSG 11 = Você tem pensado que é uma pessoa inútil? e QSG 12 = Você tem perdido o sono frequentemente por causa das suas preocupações?) que avaliam o quanto a pessoa tem experimentado os sintomas descritos, devendo ser respondidos em uma escala de quatro pontos. Para os itens negativos (por exemplo, -Tem se sentido pouco feliz e deprimido) as respostas variam de 1 (absolutamente não) a 4 (muito mais que de costume); para os itens positivos (por exemplo, -Tem se sentido capaz de tomar decisões?) as respostas variam de 1 (mais que de costume) a 4 (muito menos que de costume). Nesse sentido, os itens negativos foram invertidos, sendo a menor pontuação indicativa de melhor nível de bem-estar psicológico. Quanto menor o escore, portanto, melhor a condição de saúde do participante.

Questionário Sociodemográfico e profissional, relacionado ao vínculo com o hospital, sexo, idade, qualificação profissional, carga horaria semanal na MEJC, se possui outro vínculo empregatício, se nos últimos doze meses esteve de licença por algum motivo de saúde. 


\section{Procedimento na administração do questionário e ética da pesquisa}

Todos os procedimentos adotados nesta pesquisa seguiram as orientações previstas na Resolução 196/96 do Conselho Nacional de Saúde (CNS) e na Resolução 016/2000 do Conselho Federal de Psicologia (CNS, 1996; CFP, 2000; ANPEPP, 2000). O estudo foi submetido ao conselho de ética e aprovado sob o CAAE: 83102917.2.0000.5296.

Após aprovação pelo Comitê de Ética, os instrumentos foram encaminhados aos respondentes através de um formulário eletrônico disponível on-line na página do GoogleDocs durante um período de trinta dias. Ao solicitar a participação dos enfermeiros, foi informado aos respondentes que o objetivo do estudo seria o de avaliar a percepção dos profissionais em relação ao ambiente de trabalho e a influência deste na sua saúde geral. Aos enfermeiros foi solicitada a participação voluntária, e estes assinaram o Termo de Consentimento Livre e Esclarecido (TCLE), destacando na parte eletrônica a sua autorização.

As pessoas que se mostraram interessadas e deram o seu consentimento em participar da amostra do estudo foram esclarecidas quanto as suas respostas serem pessoais e sem interferências do administrador da pesquisa. Ao responder o instrumento, não haveria respostas certas ou erradas, com estas tratadas de acordo com o que o sujeito pensou ao ler as questões apresentadas, indicando a sua resposta no instrumento apresentado. Foi assegurado o anonimato das respostas, bem como foi informado que as questões respondidas seriam tratadas em seu conjunto de resposta e não na particularidade da resposta de cada sujeito. Apesar de ser possível encontrar as instruções necessárias para que o questionário viesse a ser respondido, o pesquisador (com experiência prévia na pesquisa) se colocou a disposição para esclarecimento sobre as dúvidas que surgissem. Um tempo médio de 20 minutos foi suficiente para que a atividade pudesse ser concluída. 


\section{Análise de dados}

Quanto à análise dos dados, utilizou-se o pacote estatístico SPSSWIN, em sua versão 24.0, para tabular os dados e realizar as análises estatísticas descritivas (média e desvio padrão, mediana), alfa de Crombach e Análise de modelagem estrutural. Para verificar a proposta do modelo teórico hipotetizado, no programa AMOS Grafics 22.0, avaliaram-se os indicadores estatísticos para o Modelo de Equações Estruturais (MEE) e foram considerados segundo a adequação de ajuste subjetiva. Esse programa estatístico tem a função de apresentar, de forma mais robusta, indicadores psicométricos que visem uma melhor construção da adaptação e acurácia da escala desenvolvida, bem como permite desenhar um modelo teórico pretendido.

Sendo um tipo de análise estatística mais criteriosa e rigorosa, testou-se o modelo teórico hipotetizado considerando alguns índices que permitam avaliar a qualidade de ajuste do próprio modelo (Hair, Tatham, Anderson, \& Black, 2005; Van de Vijver \& Leung, 1997): O X² (qui-quadrado) testa a probabilidade do modelo teórico se ajustar aos dados: quanto maior o valor do $\chi^{2}$ pior o ajustamento. Entretanto, ele tem sido pouco empregado na literatura, sendo mais comum considerar sua razão em relação aos graus de liberdade ( $\left.\chi^{2} / g .1.\right)$. Neste caso, valores até 3 indicam um ajustamento adequado; O Goodness-of-Fit Index (GFI) e o Adjusted Goodness-of-Fit Index (AGFI) são análogos ao $\mathrm{R}^{2}$ na regressão múltipla e, portanto, indicam a proporção de variância-covariância nos dados explicada pelo modelo. Os valores desses indicadores variam de o a 1, sendo que os valores na casa dos 0,80 e 0,90, ou superiores, indicam um ajustamento satisfatório; A Root-Mean-Square Error of Approximation (RMSEA), com seu intervalo de confiança de 90\% (IC90\%), é considerado um indicador de "bondade" de ajuste, isto é, valores altos indicam um modelo não ajustado. Assume-se como ideal que o RMSEA se situe entre 0,05 e 0,08, aceitando-se valores até o,10; O Comparative Fit Index (CFI) compara de forma geral o modelo estimado ao modelo nulo, considerando valores mais próximos de um como indicadores de ajustamento satisfatório; Root-Mean-Square Error of Approximation (RMSEA), com seu intervalo de confiança de 90\% 
(IC90\%), é considerado um indicador de adequação de ajuste, isto é, valores altos indicam um modelo não ajustado. Assume-se como ideal que o RMSEA se situe entre 0,05 e 0,08, aceitando-se valores de até 0,10.

\section{RESULTADOS}

Com base nos objetivos do estudo, verificou-se, inicialmente, os dados omissos, sendo menor do que $5 \%$; sendo assim, caso fossem identificados resultados "missing" ou duplicados, substituía-se pela média ou moda. Avaliou-se também, a multicolineariedade entre as variáveis, observando correlações dentro dos parâmetros (isto é, r 0,90), variando de 0,46 a o,82, permitindo gerar modelo empírico com baixo erro (Tabachnick \& Fidell, 2001). Quanto aos outliers multivariados na amostra, realizou-se o teste de normalidade de Kolmogorov-Smirnov (KS); este é empregado na análise de amostras superiores a 100 sujeitos, observando o $\mathrm{KS}=1,67, \mathrm{p}<0,18$, garantindo a normalidade (Nascimento, Tibana, Melo \& Prestes, 2015).

A partir desses resultados, avaliou-se a consistência interna das medidas utilizadas. Tal condição foi devido à observação dos seguintes pontos: tempo de publicação das escalas, pois já passavam dos cinco anos de sua publicação; a característica da amostra, já que, em uma pesquisa realizada nos sites de busca da produção científica, não foram encontrados estudos que utilizassem essas escalas com enfermeiros; procurou-se verificar se os indicadores de consistência das medidas se mantinham de acordo com o que eram apresentados pelos autores em estudos anteriores (Formiga, Fleury, \& Souza, 2014; Formiga, Viseu, \& Jesus, 2014; Pasquali et al., 1994). Como critério da avaliação da consistência, realizou-se o cálculo do Alfa de Cronbach e da correlação intraclasse (ICC), observando que a escala da Qualidade de Saúde Geral (QSG) apresentou um alfa de ( $\alpha$ ) o,86 (ICC = 0,85, [95\%IC] = 0,81-0,89), a Escala de Capital Psicológico Positivo (ECPP) teve alfa de $0,87(\mathrm{ICC}=0,88,[95 \% \mathrm{IC}]=0,84-0,91)$ e a Escala de Percepção do Suporte Organizacional (EPSO), um alfa de 0,73 (ICC = 0,73, [95\%IC] = 0,67-0,74). Estas medidas tanto estiveram acima dos escores alfas esperados $(\alpha \geq 0,70)$ quanto foram consideradas significativas. Chama atenção o resultado do intervalo de confiança no Coeficiente de Correlação Intraclasse 
(CCI), o qual permaneceu em intervalos próximos aos observados no alfa de Cronbach $(\alpha)$, garantindo com isso a confiabilidade da medida na amostra estudada (Pasquali, 2011; Kline, 2014).

Tendo confirmado que tais medidas foram confiáveis para avaliação do comportamento organizacional e da saúde geral dos respondentes, procurou-se atender ao objetivo principal do estudo (verificar a relação entre suporte organizacional, saúde geral e capital psicológico em enfermeiros). Considerando um modelo recursivo de equações estruturais, efetuaram-se os cálculos a fim de verificar o modelo hipotetizado. Gerada a análise e realizadas as devidas modificações nos ajustes de erro, o modelo proposto apresentou a seguinte razão estatística: $X^{2} / \mathrm{gl}=1,20, \mathrm{GFI}=0,83$; AGFI $=0,77$; CFI $=0,95$, TLI $=0,80$, RMSEA $=0,05(0,03-0,06) .0$ peso da variável do suporte organizacional associou-se positivamente ao QSG $(\lambda=0,09)$ e ao capital psicológico positivo $(\lambda=0,32)$, com as duas últimas variáveis se relacionando positivamente entre si $(\lambda=0,63)$ (Figura 1).

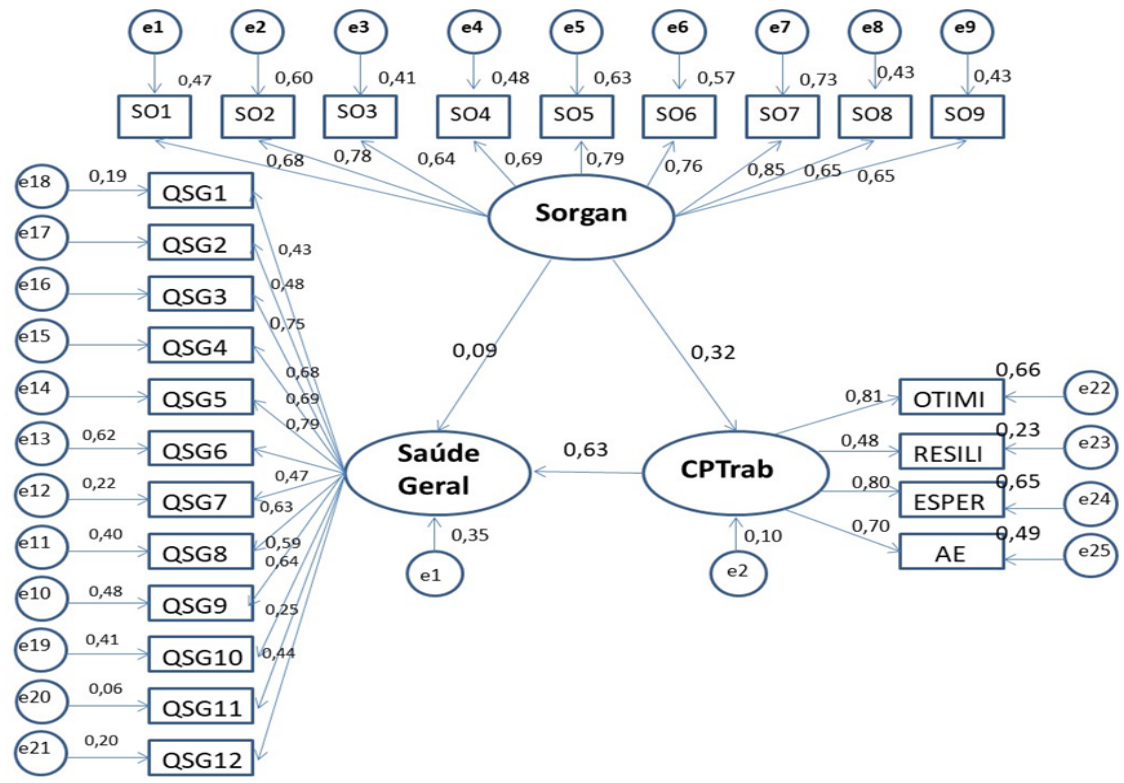

Figura 1 - Representação gráfica do modelo teórico hipotetizado

Além de os indicadores estatísticos (por exemplo, $X^{2} / \mathrm{gl}$, GFI, AGFI, CFI, TLI e RMSEA), em seu conjunto, não atenderem totalmente 
aos critérios estatísticos para que a hipótese fosse confirmada, pois os seus escores estiveram abaixo do que é exigido pela literatura estatística, a saturação do escore mínimo do Lambda $(\lambda)$ não foi maior do que 0,30 , bem como observou-se a sua condição de não-significância (Van De Vijver \& Leung, 1997; Hair et al., 2005). Mesmo observando que os Lambdas estiveram no intervalo esperado $|0-1|$ na avaliação das estimativas de predição entre as associações destas variáveis, a qual tem por base a análise de regressão referente ao modelo teórico elaborado, destaca-se que, na associação entre o suporte organizacional e QSG, não houve resultado significativo, com $\mathrm{p}<0,05$ (observou-se um $\mathrm{p}<\mathrm{0,39}$ ). Com base nestas condições, foi proposto um novo modelo teórico, excluindo a hipótese inicial relativa a uma associação interdependente entre as três variáveis.

Gerou-se um modelo alternativo hipotetizando a possibilidade de existir uma associação mediacional entre as variáveis; assim, questiona-se: existiria uma associação do suporte organizacional com o capital psicológico e deste com a saúde geral em enfermeiros? Com base no modelo não recursivo, visando a verificação desta nova hipótese, observou-se, a partir modificações nos ajustes de erro, que o pretenso modelo teórico revelou a seguinte razão estatística: $X^{2} / \mathrm{gl}=1,21, \mathrm{GFI}=0,91$; AGFI = 0,89; $\mathrm{CFI}=0,96$, TLI $=0,95$, RMSEA $=0,04(0,02-0,06)$. Com isso, o escore da variável do suporte organizacional associou-se positivamente ao capital psicológico positivo $(\lambda=0,31)$ e este, ao QSG $(\lambda=0,59)$. Não apenas houve saturação (Lambdas, $\lambda$ ) dentro do intervalo $|0-1|$ (Figura 2), mas estas também foram significativas (Tabela 1). 


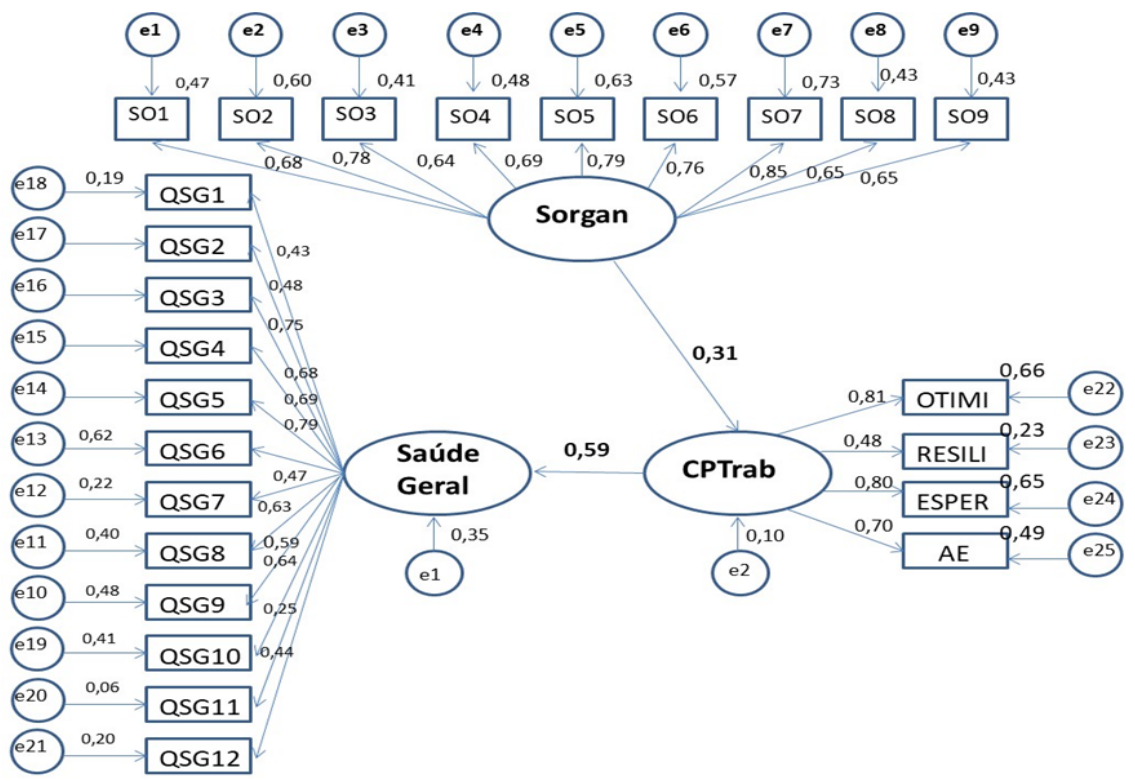

Figura 2 - Representação gráfica do modelo teórico alternativo.

Outra informação que confirmou o modelo alternativo foi observada quando se consultou as estimativas de predição entre as associações das variáveis, tendo todas sido significativas, com p<0,001 (Tabela 1). Comparando os indicadores psicométricos do modelo alternativo em relação aos do primeiro modelo, é possível destacar que ele apresentou melhores resultados, podendo afirmar que o modelo mediacional é melhor do que um modelo teórico sistêmico (no qual todas as variáveis estão inter-relacionadas). 


\section{Tabela 1 - Indicadores das estimativas preditivas entre as variáveis do modelo}

\begin{tabular}{|c|c|c|c|c|c|c|}
\hline Variável & Relação & Construto & Estimativa & d.p. & $\begin{array}{c}\text { Razão } \\
\text { Critério }\end{array}$ & $\mathrm{p}<$ \\
\hline CPTrab & $<---$ & Sorgan & 0,68 & 0,26 & 2,61 & 0,001 \\
\hline Saude_Geral & $<---$ & CPTrab & 0,20 & 0,04 & 4,63 & 0,001 \\
\hline S01 & $<---$ & Sorgan & 1,00 & --- & --- & --- \\
\hline $\mathrm{SO} 2$ & $<---$ & Sorgan & 1,24 & 0,15 & 7,93 & 0,001 \\
\hline $\mathrm{SO} 3$ & $<---$ & Sorgan & 1,03 & 0,16 & 6,09 & 0,001 \\
\hline SO4 & $<---$ & Sorgan & 1,21 & 0,20 & 5,91 & 0,001 \\
\hline SO5 & $<---$ & Sorgan & 1,18 & 0,18 & 6,33 & 0,001 \\
\hline SO6 & $<---$ & Sorgan & 1,21 & 0,19 & 6,38 & 0,001 \\
\hline SO7 & $<---$ & Sorgan & 1,46 & 0,21 & 6,83 & 0,001 \\
\hline SO8 & $<---$ & Sorgan & $-0,94$ & 0,16 & $-5,66$ & 0,001 \\
\hline SO9 & $<---$ & Sorgan & $-0,94$ & 0,18 & $-5,21$ & 0,001 \\
\hline OSG9 & $<---$ & Saude_Geral & 1,00 & --- & --- & --- \\
\hline QSG8 & $<---$ & Saude_Geral & 0,87 & 0,16 & 5,33 & 0,001 \\
\hline QSG7 & $<---$ & Saude_Geral & 0,55 & 0,16 & 3,47 & 0,001 \\
\hline QSG6 & $<---$ & Saude_Geral & 0,93 & 0,13 & 6,86 & 0,001 \\
\hline QSG5 & $<---$ & Saude_Geral & 0,75 & 0,12 & 6,08 & 0,001 \\
\hline QSG4 & $<---$ & Saude_Geral & 0,73 & 0,12 & 5,87 & 0,001 \\
\hline QSG3 & $<---$ & Saude_Geral & 0,84 & 0,12 & 6,52 & 0,001 \\
\hline OSG2 & $<---$ & Saude_Geral & 0,53 & 0,11 & 4,54 & 0,001 \\
\hline QSG1 & $<---$ & Saude_Geral & 0,43 & 0,10 & 4,05 & 0,001 \\
\hline QSG10 & $<---$ & Saude_Geral & 0,85 & 0,11 & 7,22 & 0,001 \\
\hline QSG11 & $<---$ & Saude_Geral & 0,29 & 0,13 & 2,21 & 0,001 \\
\hline QSG12 & $<---$ & Saude_Geral & 0,70 & 0,16 & 4,27 & 0,001 \\
\hline OTIM & $<---$ & CPTrab & 1,00 & --- & --- & --- \\
\hline RESIL & $<---$ & CPTrab & 0,88 & 0,17 & 4,99 & 0,001 \\
\hline ESPER & $<---$ & CPTrab & 1,92 & 0,24 & 7,82 & 0,001 \\
\hline$A E$ & $<---$ & CPTrab & 1,46 & 0,19 & 7,38 & 0,001 \\
\hline
\end{tabular}

Notas: Sorgan $=$ Suporte organizacional; $\mathrm{CPTrab}=$ Capital Psicológico no Trabalho; OTIM = Otimismo; RESIL = Resiliência $;$ ESPER = Esperança; AE = Autoeficácia.

Tendo garantido que o modelo teórico mediacional apresentou indicadores psicométricos confiáveis, procurou-se avaliar um modelo mediador, com a variável do capital psicológico intermediando o suporte 
organizacional e a saúde geral do trabalhador, pois foi mais consistente teórica e empiricamente. Buscando garantir a consistência dos resultados do modelo teórico proposto, bem como avaliar o efeito da variável mediacional, efetuou-se o teste de Sobel (z). Neste tipo de estatística, é avaliada a diferença e/ou influência das variáveis mediadoras e/ou moderadoras e o quanto estas influenciam as demais variáveis. Com isso, verifica-se o nível de mediação direta e indireta entre as variáveis independente e a dependente (Baron \& Kenny, 1986). De acordo com Baron e Kenny (1986), a variável mediadora (VMe) é uma terceira variável que deve predizer a variável dependente (VD), com a variável independente (VI) sendo preditora da variável mediadora. Na presença das duas variáveis - VI e VMe uma relação significativa prévia entre a VI e a VD decresce em magnitude, demonstrando o efeito mediacional. O modelo é caracterizado por uma relação que altera o escore para mais ou para menos a influência da VI sobre a VD. Um modelo mediacional explica as diferentes maneiras pelas quais a VI influencia a VD, encontrando uma relação direta entre VI e VD, assim como uma relação mediada por uma terceira variável, influenciando indiretamente a VD (Formiga, 2012).

Observou-se à existência de um modelo mediacional, o qual sugere que o suporte organizacional (VI, 'a') influenciou positivamente o Capital Psicológico (VMe), tendo este influenciado o QSG (VD, 'b’) ( $z=7,72$; $\mathrm{p}<\mathrm{0}, 001)$. Considerando estes resultados, o suporte organizacional influência muito mais a QSG quando é mediado pelo Capital Psicológico. As direções dos caminhos entre as variáveis destacam que o efeito preditivo é maior a partir do caminho de explicação indireta $\left(\mathrm{R}^{2}=0,13\right)$ do que do caminho direto $\left(\mathrm{R}^{2}=0,04\right)$ [95\% IC $\left.=0,42-0,71 ; \mathrm{p}<0,001\right] . \mathrm{Na}$ figura 1 , é possivel observar que, no caminho $\mathrm{a} \rightarrow \mathrm{b}$, com VMe, o Beta $(\beta)$ foi até menor, mas influenciou com base na mediação o escore da relação $\mathrm{VI} \rightarrow$ VD (c), podendo com isso observar aumento no escore $\mathrm{c}^{\prime}$ (de 0,09 para 0,25) (Figura 3). 


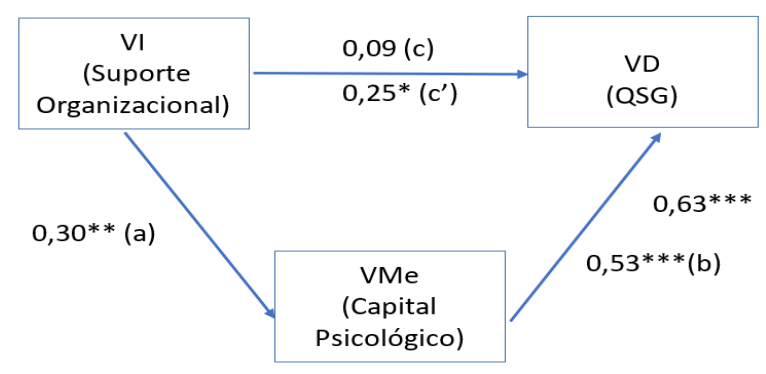

Figura 3 - Modelo mediacional para explicação da relação do suporte organizacional, por meio do capital psicológico, com a saúde geral do trabalhador.

\section{DISCUSSÃO}

Os achados neste estudo não somente comprovam que as escalas utilizadas tiveram indicadores psicométricos que confirmaram a qualidade dos construtos administrados aos profissionais de enfermagem, mas, também, que elas são consistentes. É possível afirmar que os respondentes foram capazes de avaliar a percepção de suporte organizacional, capital psicológico positivo e saúde geral de forma fidedigna. Assim, em estudos futuros, é possível administrar, com base no coeficiente CCI (Intervalos intraclasse), os mesmos instrumentos em nova amostra de enfermeiros, pois espera-se que eles respondam em direção semelhante ao que foi proposto nos construtos avaliados nesta pesquisa.

Quanto ao modelo teórico que se esperava, considera-se que ele foi refutado, o que permitiu gerar um modelo alternativo, que, por sua vez, foi confirmado. A importância deste modelo não se refere somente a sua garantia psicométrica, mas sugere uma crítica teórica e empírica à associação entre tais variáveis: pauta-se na atribuição exclusiva de que a organização é responsável pela saúde do trabalhador, tornando assim, o RH e a política de segurança no trabalho como o instrumento organizacional único na promoção de atitudes saudáveis do trabalhador. O fato principal deste modelo é sugerir uma condição de que a empresa não deverá ser, por si só, fator de suficiência para a promoção da saúde laboral; é preciso 
também que o profissional apresente engajamento e envolvimento organizacional na busca de instrumentos e eventos que favoreçam a qualidade de vida social e laboral.

As reflexões apresentadas, a partir desses resultados, são confirmados por Azambuja, Pires, Vaz e Mariale (2010), os quais afirmam que os trabalhadores envolvidos por um fazer mecanicista, na maioria das vezes, estão mais preocupados em cumprir o que lhe foi determinado, deixando de lado a reflexão sobre o seu próprio trabalho e o que pode ser trabalhado e desenvolvido internamente, com vistas a um melhor desempenho e à qualidade de vida no trabalho. Na direção deste mesmo raciocínio, contemplando distintos construtos, Elias e Navarro (2006), Esboriol (2013) e Alevato (2016) observam que os sujeitos na área de saúde que apresentam maior habilidade na inteligência emocional e no capital psicológico positivo demonstram um melhor desempenho no trabalho e confiança profissional. A probabilidade de contribuir com os objetivos da empresa e superar conflitos e obstáculos na sua funcionalidade laboral é bem maior quando inclui a percepção de suporte organizacional. Este construto permite que o trabalhador desenvolva uma maior capacidade na empresa que lhe apoia; assim, provavelmente, apresentará melhor êxito nas tarefas exigidas pelos gestores (Lucas, 2015).

O modelo alternativo estabelece um caminho relacional de mediação, sugerindo que, para verificar o papel da organização na saúde do trabalhador, é preciso, também, que o funcionário estabeleça um estado psicológico positivo. Este estado poderá até ter um apoio da própria organização, mas, ao focar nos achados do estudo, considera-se que é de responsabilidade do trabalhador o desenvolvimento dessa experiência, pois conduzirá a si e ao seu entorno laboral para condições propícias à saúde em geral.

\section{CONSIDERAÇÕES FINAIS}

Os constructos utilizados sobre o suporte organizacional e o capital psicológico no trabalho, quando aplicados às organizações e ao ambiente laboral, são capazes de potencializar a elaboração e a implementação de organizações mais saudáveis, as quais, aliadas aos estudos sobre saúde do 
trabalhador, tornam-se fatores transformadores da realidade social e da gestão positiva das organizações (Silva, Boehs, \& Cugnier, 2017). O capital psicológico no trabalho não é uma variável a mais, trata-se de um elemento do evento humano, compreendido como uma espécie de investimento emocional (comparável ao investimento econômico) para que o trabalhador não seja surpreendido com os reveses econômico, social e político na construção da sociedade e da organização. Este construto poderá interferir na qualidade do equilíbrio afetivo e inibir numa expectativa de futuro se, em algum momento, sofrer uma pressão profissional por parte do mercado ou da empresa e/ou dinâmica na mudança profissional (por exemplo, desemprego, alteração de função, compartilhamento de funções com funcionários mais novos, etc.), permitindo que o trabalhador consiga projetar saídas profissionais empreendedoras e criativas (Lima, Lopes, Nassif, \& Silva, 2015a; Lima, Lopes, Nassif, \& Silva, 2015b; Formiga \& Freire, 2018).

Considera-se que o capital psicológico positivo, de acordo com Formiga, Viseu e Jesus (2014), deve ser considerado um mecanismo sócio-humano dinâmico na área organizacional, pois conduz ao enfrentamento positivo, com base em um estado emocional e comportamental menos agressivo e estressor, revelando um esforço destinado à atitude para alcançar o êxito humano-profissional na demanda dos desafios sociais e profissionais, e não simplesmente permanecer na crítica e desencanto profissional, sem ação para mudança e investimento nas novas possibilidades de empreender no ambiente de trabalho (Salessi \& Omar, 2014; Assis \& Nascimento, 2017).

Com isso, espera-se que os objetivos do estudo tenham sido cumpridos, especialmente no que se refere a verificação de um modelo que se concentre nas variáveis da psicologia positiva (isto é, variáveis mais alternativas e inovadoras na explicação da saúde do trabalhador), as quais têm sido inseridas no espaço teórico da psicologia organizacional nos últimos anos. Acredita-se que a importância desse tipo de estudo tem sua motivação na compreensão dos mecanismos de um não adoecimento. Apesar de os resultados revelarem indicadores estatísticos que contribuem para confirmar a hipótese que direcionou o estudo para uma nova perspectiva, espera-se que estudos futuros possam ser realizados com o objetivo de verificar novas associações entre variáveis divergentes e convergentes, bem 
como, com amostras semelhantes e distintas ao do presente estudo. Seria interessante um estudo que abordasse as variáveis sociodemográficas e de estrutura familiar; também seria útil um estudo intra, inter e transcultural com o objetivo de avaliar a variação entre as variáveis em distintos hospitais.

\section{REFERÊNCIAS}

Alevato, H. M. (2016). Gestão de pessoas, grupalidade e saúde no trabalho. Boletim Técnico do Senac, 31(2), 51-63.

Antunes, R. (2009). Os sentidos do trabalho: ensaio sobre a afirmação e a negação do trabalho. 2 .ed. São Paulo: Boitempo.

Assis, D. M., \& Nascimento, J. L. (2017). Cinismo organizacional: Estudo preliminar de adaptação de uma escala de medida para o contexto português. Análise Psicológica, 35(3), 383-393. https://dx.doi. org/10.14417/ap.1272

Associação Nacional de Pesquisa e Pós-Graduação em Psicologia [ANPEPP]. (2000). Contribuições para a discussão das Resoluções CNS $\mathrm{n}^{\mathrm{o}}$. 196/96 e CFP Nº 016/2000. Recuperado de http://www.anpepp. org.br/XIISimposio/Rel_ComissaoEticasobre_Res_CNS_e _CFP. pdf20oo.

Avey, J. B., Luthans, F., \& Jensen, S. M. (2009). Psychological capital: A positive resource for combating employee stress and turnover. Human Resource Management, 48(5), 677-693.

Avey, J. B., Reichard, R. J., Luthans, F., \& Mhatre, K. H. (2011). Meta-analysis of the impact of positive psychological capital on emplouee attitudes, behaviors, and performance. Human Resource Development Quarterly, 22, 127-152.

Azambuja, E. P., Pires, D. E. P., Vaz, M. R. C., \& Marziale, M. H. (2010). É possível produzir saúde no trabalho da enfermagem? Texto \& contexto - enfermagem, 19(4), 658-666. Recuperado de http://www.scielo.br/scielo.php?script=sci_arttext\&pid=So10407072010000400008\&lng=en. http://dx.doi.org/10.1590/ So104-07072010000400008. 
Barbosa, A. L. N. H. (2014). Um retrato de duas décadas do mercado de trabalho brasileiro. InEm: A. A. Camarano, A. A. (Org.). Novo regime demográfico: uma nova relação entre população e desenvolvimento? (pp. 271-313). Rio de Janeiro: Ipea. p. 271-313.

Baron, R. M., \& Kenny, D. A. (1986). The moderator-mediator variable distinction in social psychological research: Conceptual, strategic, and statistical considerations. Journal of Personality and Social Psychology, 51, 1173-1182.

Barros, N. M. G. C., \& Honório, L. C. (2015). Riscos de adoecimento no trabalho de médicos e enfermeiros em um hospital regional matogrossense. REGE-Revista de Gestão, 22(1), 21-39.

Bendassolli, P. F. (2011). Crítica às apropriações psicológicas do trabalho. Psicologia \& Sociedade, 23(1), 75-84.

Brito, J., Pena, P., Gomes, L., Souto, A., \& Pires. A. (2012). A saúde dos trabalhadores de saúde: focos, abordagens e estratégias de pesquisa. InEm: J. Machado J, A. Assunção A, (Orgs). Panorama da saúde dos trabalhadores da saúde (p. 66-105). Belo Horizonte: UFMG.

Caesens, G., Stinglhamber, F., \& Ohana, M. (2016). Perceived organizational support and well-being: a weekly study. Journal of Managerial Psychology, 31(7), 1214-1230.

Castro, M. R., \& Farias, S. N. P. (2008). A produção científica sobre riscos ocupacionais a que estão expostos os trabalhadores de enfermagem. Escola Anna Nery, 12(2), 364-369. https://dx.doi. org/10.1590/S1414-81452008000200026

Chaves, L. D., Ramos, L. H., \& Figueiredo, E. N. (2011). Job satisfaction of nurses working in Brazil. Acta Paulista de Enfermagem, 24(4), 507-513. https://dx.doi.org/10.1590/So103-21002011000400010

Chong, H., White, R. E., \& Prybutok, V. (2001). Relationship among organizational support. Industrial Management \& Data Systems, 101(6), 273-281,. https://doi.org/10.1108/EUMooooooooo5576.

Conselho Federal de Psicologia [CFP]. (2000). Resolução CFP no O16/200o de 20 de dezembro de 20oo. Dispõe sobre a realização de pesquisa em Psicologia com seres humanos. Brasília, DF. 
Conselho Nacional de Saúde [CNS]. (1996). Resolução $n^{o}$ 196, de 10 de outubro de 1996. https://bvsms.saude.gov.br/bvs/saudelegis/ cns/1996/res0196_10_10_1996.html

Csikszentmihalyi, M. (2004). Gestão qualificada: a conexão entre felicidade negócio. (1a ed.). Porto Alegre: Bookman.

Eisenberger, R., Huntington, R., Hutchison, S., \& Sowa, D. (1986). Perceived Organizational Support. Journal of Applied Psychology, 71(3), 500-507.

Elias, M. A., \& Navarro, V. L. (2006). A relação entre o trabalho, a saúde e as condições de vida: negatividade e positividade no trabalho das profissionais de enfermagem de um hospital escola. Revista LatinoAmericana de Enfermagem, 14(4), 517-525. Recuperado de http:// www.scielo.br/pdf/rlae/v14n4/v14n4ao8.pdf

Esboriol, V. (2013). Inteligência emocional, capital psicológico e percepção de suporte organizacional em gestores do segmento de saúde (Dissertação de Mestrado). Universidade Metodista de São Paulo, São Bernardo do Campo. Recuperado de http://tede.metodista.br/ jspui/handle/tede/1336.

Fabri, A. C. O. C, Carvalho, A. P., Araujo, S., Goulart, L. R, Mattos, A. M., Teixeira, H. C., \& Lana, F. C. F. (2018). Antigen-specific assessment of the immunological status of various groups in a leprosy endemic region. BMC infectious diseases, 15(218), 1-9.

Faul, F., Erdfelder, E., Lang, A. G., \& Buchner, A. (2007). G* Power 3: A flexible statistical power analysis program for the social, behavioral, and biomedical sciences. Behavior Research Methods, 39(2), 175-191.

Formiga, N. S. \& Guimarães, W. N. C. (2019). Caminhos para a rentabilidade emocional da saúde laboral: a influência do capital psicológico positivo no transtorno emocional em médicos. Beau Bassin: Mauritius: Novas Edições Acadêmicas. 
Formiga, N. S., \& Freire, B. G. O. (2018). Correlatos entre suporte organizacional e expectativa de futuro em funcionários de organizações públicas e privadas no Brasil. Recuperado em 30 Agosto de 2018, http://www.psicologia.pt/artigos/ver_artigo.php?correlatos-entresuporte-organizacional-e-expectativa-de-futuro-em-funcionarios-deorganizacoes-publicas-e-privadas-no-brasil\&codigo $=\mathrm{A} 1218$

Formiga, N. S., Freire, B. G. O, \& Fernandes, A. J. (2019). Evidência de construto, invariância fatorial e validade convergente da escala de capital social organizacional em trabalhadores brasileiros. Eureka: Revista de Psicologia, 16(2), 34-59.

Formiga, N. S., Viseu, J., \& Jesus, S. N. de. (2014). Verificação empírica de uma medida de Capital Psicológico Positivo em trabalhadores brasileiros. Eureka, 11(2), 284-300.

Formiga, N., Fleury, L. F. D. O., \& Souza, M. A. D. (2014). Evidências de validade da escala de percepção de suporte organizacional em funcionários de empresa pública e privada. Estudos Interdisciplinares em Psicologia, 5(2), 60-76.

Galindo, R. H., Feliciano, K. V. O., Lima, R. A. S., \& Souza, A. I. (2012). Síndrome de Burnout entre enfermeiros de um hospital geral da cidade do Recife. Revista da Escola de Enfermagem da USP, 46(2), 420-427. Recuperado em 30 Julho de 2018,de http://www.scielo.br/scielo. php?script=sci_arttext\&pid=Soo80-62342012000200021\&lng=en . http://dx.doi.org/10.1590/Soo80-62342012000200021.

Gomes, T. D. G. (2017). A influência da gestão de recursos humanos na motivação dos colaboradores . (Dissertação de Mestrado)., Instituto Politécnico Do Porto, PortoPortugal. Recuperado deem 20 de Maio de 2018, http://recipp.ipp.pt/handle/10400.22/10956

Gouveia, V. V., Chaves, S. S. S., Oliveira, I. C. P., Dias, M. R., Gouveia, R. S. V., \& Andrade, P. R. (2003). A utilização do QSG-12 na população geral: estudo de sua validade de construto. Psicologia: Teoria e Pesquisa, 19(3), 241-248.

Hair, J. F., Tatham, R. L., Anderson, R. E., \& Black, W. (2005). Análise Multivariada de dados. Porto Alegre: Bookman.

Kline, P. (2014). An easy guide to factor analysis. New York: Routledge. 
Leite, A. F., \& Nogueira, J. A. D. (2017). Fatores condicionantes de saúde relacionados ao trabalho de professores universitários da área da saúde: uma revisão integrativa. Revista Brasileira de Saúde Ocupacional, 42, 1-15.

Lima, E., Lopes, R. A., Nassif, V. M. J., \& Silva. D. (2015a). Ser seu próprio patrão? Aperfeiçoando-se a educação superior em empreendedorismo. $R A C$, 19(4), 419- 439. doi.org/10.1590/1982-7849rac20151296.

Lima, E., Lopes, R. M., Nassif, V., \& Silva, D. (2015b). Opportunities to improve entrepreneurship education: contributions considering Brazilian challenges. Journal of Small Business Management, 53(4), 1033-1051. doi: 10.1111/jsbm.12110

Lino, M. M., Nora, P. T., Lino, M. M., \& Furtado, M. (2012). Enfermagem do trabalho à luz da visão interdisciplinar. Saúde \& transformação social 3(1), 85-91. Recuperado em o8 de dezembro de 2020, de http://pepsic.bvsalud.org/scielo. php?script=sci_arttext\&pid=S2178-70852012000100014\&lng $=$ pt \&tlng $=$ pt.

Lorenz, V. R., Benatti, M. C. C., \& Sabino, M. O. (2010). Burnout e estresse em enfermeiros de um hospital universitário de alta complexidade. Revista Latino-Americana de Enfermagem, 18(6), 1084-1091.

Lucas, C. P. V. (2015). A promoção do capital psicológico, workengagement e saúde mental de trabalhadores (Dissertação de Mestrado). Universidade de Lisboa, Portugal. Recuperado de https://www.researchgate.net/profile/Carla_Vale_Lucas/ publication/306600607_A_promocao_do_capital_ psicologicowork_engagement_e_saude_mental_de_trabalhadores/ links/5ae59d45a6fdcc3bea96363c/A-promocao-do-capitalpsicologico-work-engagement-e-saude-mental-de-trabalhadores. pdf?origin=publication_detail

Luthans, F., Luthans, K. W., \& Luthans, B. C. (2004). Positive psychological capital: Beyond human and social capital. Journal Business Horizons, 1, 45-50.

Luthans, F., Youssef, C. M., \& Avolio, B. J. (2007). Psychological capital. New York: Oxford University Press. 
Murofuse N. T., \& Marziale M. H. P. (2005). Doenças do sistema osteomuscular em trabalhadores de enfermagem. Revista Latino-Americana de Enfermagem Rev. Latino-Am. Enfermagem; 13(3), :364-73.

Nascimento, D. C., Tibana R. A., Ferreira, G. M., \& Prestes, J. (2014) Testes de normalidade em análises estatísticas: uma orientação para praticantes em ciências da saúde e atividade física. Revista Mackenzie de Educação Física e Esporte, 14(2), 73-77.

Neves, P., \& Eisenberger, R. (2014). Perceived organizational support and risk taking. Journal of managerial psychology, 29(2), 187-205.

Oliveira-Castro, G. A., Pilati, R., \& Borges-Andrade, J. E. (1999). Percepção de suporte organizacional: desenvolvimento e validação de um questionário. Revista de Administração Contemporânea, 3(2), 29-51. Recuperado de em 02 de Novembro de 2017. https://dx.doi. org/10.1590/S1415-65551999000200003

Paschoal, T. (2008). Bem-estar no trabalho: relações com suporte organizacional, prioridades axiológicas e oportunidades de alcance de valores pessoais no trabalho (. Tese de Doutorado)., Universidade de Brasília, Brasília.

Paschoal, T., Torres, C. V., \& Porto, J. B. (2010). Felicidade no trabalho: relações com suporte organizacional e suporte social. Revista de Administração Contemporânea, 14(6), 1054-1072. Recuperado de em 02 de Agosto de 2017, http://www.scielo.br/scielo. php?script=sci_arttext\&pid=S1415-65552010000700005.

Pasquali, L. (2011). Psicometria: teoria dos testes na psicologia e na educação. 4 ed. Petrópolis: Vozes.

Pasquali, L., Gouveia, V. V., Andriola, W. B., Miranda, F. J., \& Ramos, A. L. M. (1994). Questionário de Saúde Geral de Goldberg (QSG): Adaptação brasileira. Psicologia: Teoria e Pesquisa, 1O(3), 421-438. Recuperado de https://www.researchgate.net/ publication/279191898_Questionario_de_Saude_Geral_de_ Goldberg_QSG_Adaptacao_Brasileira 
Polit, D. F., \& Beck, C. T. (2011). Fundamentos de pesquisa em enfermagem: avaliação de evidências para a prática de enfermagem. (7a eEd.). Porto Alegre: Artmed.

Salessi, S., \& Omar, A. (2014). Validation of the organizational cynicism scale: A study with Argentinean workers. Psicología, 32(2), 357-385. Recuperado de http://revistas.pucp.edu.pe/index.php/psicologia/ article/view/10953

Scorsolini-Comin, F., \& dos Santos, M. A. (2010). Psicologia Positiva e os Instrumentos de Avaliação no Contexto Brasileiro. Psicologia: Reflexão e Crítica, 23(3), 440-448.

Silva, N., Boehs, S. T. M, \& Cugnier, J. S. (2017). Psicologia positiva aplicada às organizações e ao trabalho. Em: S. T. M. Boehs, \& N. Silva (Orgs.). Psicologia Positiva nas Organizações e no Trabalho: conceitos fundamentais e sentidos aplicados (pp. 43-60). São Paulo: Vetor.

Siqueira, M. M. M. (1995). Antecedentes de comportamentos de cidadania organizacional: análise de um modelo pós-cognitivo . (Tese de doutorado Doutorado).não-publicada, Universidade de Brasília, Brasília.

Siqueira, M., M., M. (2014). Medidas do comportamento organizacional: ferramentas de diagnóstico e de gestão. Porto Alegre: Artmed.

Siqueira, M. M. M., \& Padovan, V. A. R. (2008). Bases teóricas de bem-estar subjetivo, bem-estar psicológico e bem-estar no trabalho. Psicologia: Teoria e Pesquisa, 24(2), 201-209.

Tabachnick, B., \& Fidell, L. (2001). Using multivariate statistics. Needham Heights: Allyn \& Bacon.

Tamayo, M. R., \& Tróccoli, B. T. (2002). Exaustão emocional: relações com a percepção de suporte organizacional e com as estratégias de coping no trabalho. Estudos de Psicologia,7(1), 37-46, 2002.

Torres, A. R. A., Chagas, M. I. O., Moreira, A. C. A., Barreto, I. C. D. H. C., \& Rodrigues, E. M. (2013). O adoecimento no trabalho: repercussões na vida do trabalhador e de sua família. SANARE-Revista de Políticas Públicas, 1O(1), 42-48.

Van de Vijver, F., \& Leung, K. (1997). Methods and data analysis for crosscultural research. Thousand Oaks: Sage Publications. 
Viseu, J., Jesus, S. N., Rus, C., Nunes, H., Lobo, P., \& Cara-Linda, I. (2012). Capital Psicológico e sua avaliação com o PCQ-12. ECOS-Estudos Contemporâneos da Subjetividade, 2(1), 4-16.

Zanelli, J. C., Borges-Andrade, J. E., \& Bastos, A. V. B. (2004). Psicologia, organizações e trabalho no Brasil. Porto Alegre: Artmed.

Recebido em 15/10/2019 Aceito em 30/09/2020 